\title{
Polymer Chemistry

\section{Well-defined polymeric vesicles with high stability and modulation of cell uptake by a simple coating protocol $\dagger$}

\author{
Gökçen Yaşayan, Martin Redhead, Johannes P. Magnusson, Sebastian G. Spain, Stephanie Allen, \\ Martyn Davies, Cameron Alexander* and Francisco Fernández-Trillo*
}

Received 18th May 2012, Accepted 21st June 2012

DOI: 10.1039/c2py20352a

\begin{abstract}
Amphiphilic polymers have been synthesised by controlled free radical polymerisation techniques. These polymers self-assemble into well-defined vesicles in aqueous conditions, enabling encapsulation of a model hydrophilic molecule. The polymeric vesicles show high stability against a range of aqueous conditions with marginal release of cargo, even in the presence of known cell-membrane disruptive polymers such as branched poly(ethylene imine) (b-PEI). This stability allows for inversion of the surface charge of the polymeric vesicles by a simple coating protocol leading to an enhanced uptake by mammalian cells.
\end{abstract}

\section{Introduction}

Vesicles and membranes are vital components in nature, with critical roles at the cellular level such as compartmentalisation, storage, nutrient transport or information protection. It is not surprising then that the construction of vesicles with tailored properties is of interest in fields such as drug delivery, ${ }^{1-3}$ nanoscience, ${ }^{4,5}$ and synthetic biology.${ }^{6,7}$ Biological systems have relied on phospholipids to produce vesicles and have been able to create extremely complex cellular membranes. Unfortunately, phospholipidic membranes are inherently unstable. In nature this drawback is overcome by blending with other amphiphiles such as cholesterol. In addition, natural vesicles incorporate specialised membrane proteins to ensure that the right osmotic balance is maintained to prevent vesicle rupture. While this approach generates highly functional vesicles, it is extremely difficult and costly to reproduce in the laboratory. In recent years, synthetic chemistry has expanded the number of amphiphiles that can form vesicular aggregates, with some examples showing improved stabilities when compared to phospholipidic vesicles or liposomes. ${ }^{\mathbf{8 - 1 1}}$ Among these amphiphiles, polymers offer great potential in the preparation of vesicles, as the correct choice of polymerisation techniques, allows for a wide range of physical, chemical and mechanical properties. In addition, factors such as polymer architecture and molecular weight (MW) can be controlled in order to enhance overall properties. ${ }^{12-18}$

School of Pharmacy, The University of Nottingham, University Park, Nottingham NG7 2RD, UK. E-mail: cameron.alexander@nottingham.ac. uk; francisco.fernandez-trillo@nottingham.ac.uk

$\uparrow$ Electronic supplementary information (ESI) available: Monomer synthesis, polymer characterisation, additional AFM and TEM micrographs, vesicle stability data and further cell imaging. See DOI: $10.1039 / \mathrm{c} 2 \mathrm{py} 20352 \mathrm{a}$ a)

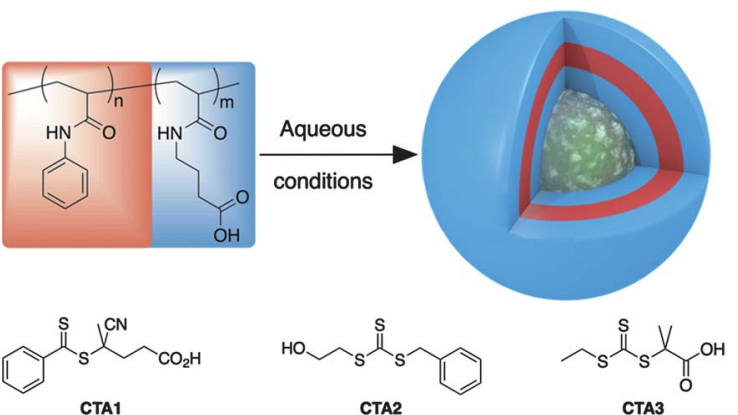

c)

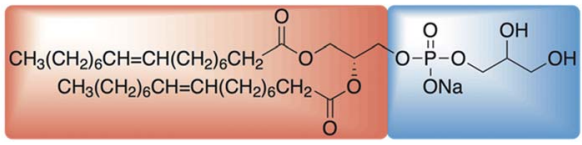

Scheme 1 (a) Schematic representation of the polymers prepared in this work and their assembly into vesicles. (b) RAFT agent used in this work. (c) Model lipid used for vesicle stability comparison.

In this paper, we describe the synthesis of well defined polymeric vesicles from novel amphiphiles based on acrylamide polymers (Scheme 1). In addition, the stability of these polymersomes against different conditions, such as increased $\mathrm{pH}$ and buffer strength is evaluated and compared to model conventional liposomes. Biocompatibility and cellular uptake for these materials is also investigated.

\section{Materials and methods}

Phenylacrylamide (PAm) and $N$ - $\omega$-acrylamidobutanoic acid (4AmBA) were prepared according to a modified literature procedure $(\mathrm{ESI} \dagger) .{ }^{19}$ 4-Cyano-4-((thiobenzoyl)sulfanyl)pentanoic acid 
(CTA1), benzyl 2-hydroxyethyl carbonotrithioate (CTA2) and 2(ethylthiocarbonothioylthio)-2-methylpropanoic acid (CTA3) were prepared according to literature procedures. ${ }^{20,21} 4,4^{\prime}$-azobis(4-cyanopentanoic acid) (V-501) was purchased from Fluka ${ }^{\circledR}$ and recrystallised from $\mathrm{MeOH}$. Branched polyethyleneimine (b-PEI) (average $M_{\mathrm{w}}: \sim 25000$ (LS)) was purchased from Aldrich ${ }^{\circledR}$. All other chemicals were purchased from Sigma-Aldrich ${ }^{\circledR}$ and used without further purification. Dulbecco's Phosphate Buffered Saline (DPBS) was purchased from Lonza Group Ltd. All other solvents were HPLC grade, purchased from Sigma-Aldrich ${ }^{\circledR}$ or Fisher Scientific $\AA$, and used without further purification. Mica discs and specimen discs were purchased from Agar Scientific Ltd. ScanAsyst-Fluid+ AFM probes (resonant mechanical frequency: 120$180 \mathrm{kHz}$, spring constant: $0.7 \mathrm{~N} \mathrm{~m}^{-1}$ ) were purchased from Bruker®. Syringe filters $(0.2 \mu \mathrm{m}$ and $0.45 \mu \mathrm{m})$ were purchased from Millipore $^{\mathrm{TM}}$. Disposable capillary cells for zeta potential measurements were purchased from Malvern Instruments. Copper grids coated with a formvar carbon film were purchased from TAAB Laboratories Equipment Ltd.

Nuclear Magnetic Resonance (NMR) spectra were recorded on a Bruker $400 \mathrm{MHz}$ spectrometer. Chemical shifts are reported in $\operatorname{ppm}$ ( $\delta$ units) downfield from internal tetramethylsilane. Mass spectra (MS) (TOF-ESI) were recorded on a Waters 2795 separation module/micromass $\mathrm{LCT}$ platform, under negative scan mode with direct injection of the purified compounds. Gel Permeation Chromatography (GPC) was carried out using Polymer Laboratories GPC 50 with RI detector. DPBS or DMF/ $0.1 \% \mathrm{w} / \mathrm{v} \mathrm{LiBr}$ were used as the mobile phase. Molecular weights were calculated based on a standard calibration method using poly(ethylene glycol) (DPBS) or poly(methyl methacrylate) $(\mathrm{DMF} / \mathrm{LiBr})$ narrow standards. Fluorescence spectra were recorded on a Cary Eclipse fluorescence spectrometer (Agilent Technologies). Dynamic Light Scattering (DLS) was measured using a Viscotec Model 802 instrument equipped with an internal laser $(825-832 \mathrm{~nm})$ with a maximum radiation power of $60 \mathrm{~mW}$. Atomic Force Microscopy (AFM) topography images and particle analysis of block copolymer vesicles were obtained in liquid at room temperature using a Multimode 8 Scanning Probe Microscopy station, operating in PeakForce Tapping ${ }^{\mathrm{TM}}$ mode under ScanAsyst auto control. Images were acquired using an Escanner, at scan rates between 1 and $3 \mathrm{~Hz}$, with a resolution of $512 \times 512$ pixels. Transmission Electron Microscopy (TEM) was performed on a FEI Tecnai ${ }^{\mathrm{TM}} 12$ Biotwin transmission electron microscope. Zeta potential was measured using a Zetasizer Nano ZS (Malvern Instruments Ltd) at $25^{\circ} \mathrm{C}$.

NIH 3T3 Fibroblasts were cultured between passages 15-20, A549 cells were cultured from passage 6. Media for the two cell lines was MEM, supplemented with 10\% FBS, 2 mM L-glutamine, 1000 au $\mathrm{mL}^{-1}$ penicillin and $10 \mu \mathrm{g} \mathrm{mL}^{-1}$ streptomycin. Cells were cultured at $37{ }^{\circ} \mathrm{C}$ and $5 \% \mathrm{CO}_{2}$ in a humidified atmosphere. Images were obtained using a Leica DM IRB microscope with a QICAM FAST1394 camera (QImaging, Surrey Canada). Image analysis was performed using Origin 8 pro (Origin Labs, UK). Acute cytotoxicity was assessed using CytotoxONE ${ }^{\mathrm{TM}}$ assay kit (Promega Corporation, UK), chronic toxicity was measured using $1 \mathrm{mg} \mathrm{mL}^{-1}$ MTT reagent. Absorbance and fluorescence measurements were obtained using a TECAN colorimetric/fluorimetric plate reader (TECAN, Männedorf, Switzerland).
General protocol for reversible addition-fragmentation chain transfer (RAFT) polymerisation

Polymerisations were conducted in round bottom flasks sealed with a rubber septum and parafilm. An NMR spectrum was recorded at the beginning of the experiment. The polymerisation solutions were degassed using argon for at least $10 \mathrm{~min}$ and transferred to an oil bath preheated to $70{ }^{\circ} \mathrm{C}$. To stop the polymerisation, the solution was quenched by cooling in ice-water and opening to air, and another NMR spectrum was recorded to enable calculation of degree of conversion. For the removal of the RAFT agent in the final block copolymers, the reaction was carried out at $80^{\circ} \mathrm{C}$.

\section{Polymer conversion and composition calculations}

The overall monomer conversion was calculated by ${ }^{1} \mathrm{H}$ NMR spectra by comparing the vinyl proton signals from the monomers $(5.6$ and $6.1 \mathrm{ppm})$ to the overall integration from the amide hydrogens (7.5-8.2 ppm) in the case of $\mathbf{A A m B A}$, and to the overall integration of the aromatic groups (7.0-7.7 ppm) for PAm and the block copolymers.

$\operatorname{Poly}(N$-phenylacrylamide) (p(PAm)-R) (P1). In a typical experiment, to a solution of PAm (3012 mg, $20.5 \mathrm{mmol}, 1.61 \mathrm{M}$ ) in DMF (12.7 mL), CTA1 (116 mg, $0.415 \mathrm{mmol})$ and V-501 (29.0 $\mathrm{mg}, 0.106 \mathrm{mmol}$ ) were added. The polymerisation was carried out for $15 \mathrm{~h} 44 \mathrm{~min}$ ( $85 \%$ conversion). The title compound $\mathbf{P 1}$ was purified by precipitation in $\mathrm{Et}_{2} \mathrm{O}$ and recovered as a light orange powder (2370 mg, 89\% yield) after drying under vacuum (dark, 2 days). $M_{\mathrm{w}}$ (GPC) 13 127, PDI (GPC) 1.10.

Poly(4-acrylamidobutanoic acid) (p(4AmBA)-R) (P2). In a typical experiment, 4AmBA (499 mg, $3.17 \mathrm{mmol}, 0.91 \mathrm{M}$ ) in $\mathrm{H}_{2} \mathrm{O}$ (3.50 mL), CTA3 (23.8 mg, $0.106 \mathrm{mmol}, 0.44 \mathrm{M})$ in EtOH $(0.440 \mathrm{~mL})$ and $\mathbf{V - 5 0 1}(2.88 \mathrm{mg}, 0.010 \mathrm{mmol}, 0.03 \mathrm{M})$ in EtOH $(0.300 \mathrm{~mL})$ were prepared separately and then mixed together, to make a final $0.75 \mathrm{M}$ solution of 4AmBA. The polymerisation was carried out for $3 \mathrm{~h}$ ( $92 \%$ conversion). The title compound P2 was purified by dialysis against water and recovered as a yellow powder (364 mg, 75\% yield) after freeze-drying from water (dark, 2 days). $M_{\mathrm{w}}$ (GPC) 3492, PDI (GPC) 1.09.

Poly(4-acrylamidobutanoic acid)-block-poly $(N$-phenylacrylamide)-RAFT (p(4AmBA)-b-p(PAm)-R). In a typical experiment, PAm (298 mg, $2.03 \mathrm{mmol}, 0.53 \mathrm{M})$ in DMSO (3.80 mL), P2 (100 $\mathrm{mg}, 16.4 \mu \mathrm{mol})$ and a solution of $\mathbf{V - 5 0 1}(0.175 \mathrm{~mL}, 1.54 \mathrm{mg}, 5.49$ $\mu \mathrm{mol}, 3.14 \mathrm{mM})$ in EtOH $(0.300 \mathrm{~mL})$ were added, to make a final $0.50 \mathrm{M}$ solution of PAm. The polymerisation was carried out for 1 h 20 m (85\% conversion). The title compound p(4AmBA)-b$\mathbf{p}(\mathbf{P A m})-\mathbf{R}$ was purified by precipitation into EtOAc and recovered as a light yellow powder (283 mg, 71\% yield) after freezedrying from water (dark, 2 days).

Poly(4-acrylamidobutanoic acid)-block-poly( $N$-phenylacrylamide) (p(4AmBA)-b-p(PAm)) (P3). In a typical experiment, p(4AmBA)-b-p(PAm)-R (250 mg, $\left.11.6 \mu \mathrm{mol}, 50 \mathrm{mg} \mathrm{mL}^{-1}\right)$ in DMSO $(5 \mathrm{~mL})$, AIBN (95 mg, $57.3 \mu \mathrm{mol})$ was added. The reaction was carried out overnight. The title compound $\mathbf{P 3}$ was purified by precipitation into EtOAc : $\mathrm{Et}_{2} \mathrm{O} 1: 1$ and recovered 
as a white powder (250 mg, quantitative yield) after freeze-drying from water (dark, 2 days).

\section{General protocol for vesicle preparation}

A solution of polymer $(5-15 \mathrm{mg})$ in $1 \mathrm{~mL}$ of THF/MeOH $(9: 1)$ was added drop-wise onto $1 \mathrm{~mL}$ of buffer (10 mM HEPES, $10 \mathrm{mM} \mathrm{NaCl}, \mathrm{pH} \mathrm{7.4)}$, and the organic solvent was allowed to evaporate overnight. The resulting suspension was subjected to 8 freeze-thaw cycles (liquid $\mathrm{N}_{2}, 40{ }^{\circ} \mathrm{C}$ water bath), and dialysed against osmotic buffer (10 mM HEPES, $100 \mathrm{mM} \mathrm{NaCl}$, pH 10 and $7.4,2$ days).

\section{Dye encapsulation assay}

$20 \mu \mathrm{L}$ of polymeric vesicles solution prepared as described above, containing $50 \mathrm{mM}$ 5(6)-carboxyfluorescein (CF), were diluted up to $1 \mathrm{~mL}$ with additional osmotic buffer (10 mM HEPES, $100 \mathrm{mM}$ $\mathrm{NaCl}, \mathrm{pH}$ 7.4). The change in fluorescence intensity $\left(I_{\mathrm{t}}\right)$ from $500 \mathrm{~nm}$ to $600 \mathrm{~nm}$ was monitored $\left(\lambda_{\mathrm{exc}}=492 \mathrm{~nm}, \lambda_{\mathrm{em}}=517 \mathrm{~nm}\right)$ before and after disruption of the vesicle membrane by addition of $20 \mu \mathrm{L}$ of Triton $\mathrm{X}-100\left(20 \% \mathrm{w} / \mathrm{w}\right.$ in $\left.\mathrm{H}_{2} \mathrm{O}\right)$.

\section{General protocol for coating vesicles with b-PEI}

$15 \mathrm{mg} \mathrm{mL}^{-1}$ polymeric vesicle solutions prepared as described before and a $20 \mathrm{mg} \mathrm{mL}^{-1}$ solution of b-PEI were mixed with a polymer : b-PEI ratio of $1: 2$, under stirring, and the system allowed to stand for $15 \mathrm{~min}$. Excess of b-PEI was removed filtering through Sephadex G-25.

\section{DLS}

Polymeric vesicles were prepared as described above and diluted at least 10 times with additional osmotic buffer prior to DLS analysis. Hydrodynamic radii $\left(R_{\mathrm{H}}\right)$ of the polymeric vesicles were measured via scattered light recorded at a $90^{\circ}$ angle to incident radiation. Data processing was performed with the OmniSize 3.0 software. From standard auto correlation functions, measured diffusion coefficients were related to particle $R_{\mathrm{H}}$ via the StokesEinstein equation (eqn (1))

$$
R_{\mathrm{H}}=k T / 6 \pi \eta D
$$

where $R_{\mathrm{H}}$ is the hydrodynamic radius, $k$ is the Boltzmann constant, $T$ is the temperature, $\eta$ is the viscosity of the solvent and $D$ the diffusion constant. It was assumed that particles were spherical and non-interacting, and the viscosity of the buffer equal to PBS. Measurements quoted are the averages of at least 10 readings of particle size recorded at $25^{\circ} \mathrm{C}$.

\section{AFM}

$10 \mathrm{mM} \mathrm{MgCl} 2$ solution was incubated with freshly cleaved mica for $10 \mathrm{~min}$, and then the mica was washed with distilled water several times and blown dry completely with nitrogen at room temperature. Polymeric vesicles were prepared as described above and filtered through $0.2 \mu \mathrm{m}$ syringe filter, and diluted with additional osmotic buffer (10 mM HEPES, $100 \mathrm{mM} \mathrm{NaCl}, \mathrm{pH}$ 7.4), to a final concentration of $500 \mu \mathrm{g} \mathrm{mL}^{-1}$ and filtered through a $0.45 \mu \mathrm{m}$ syringe filter. Measurements quoted are the averages of at least 50 particles recorded at $25^{\circ} \mathrm{C}$. Image data were analysed using NanoScope Analysis software (Version 1.20 (Bruker)). For b-PEI coated vesicles, freshly cleaved mica without $\mathrm{MgCl}_{2}$ pretreatment was employed.

\section{TEM}

Polymeric vesicles prepared as described above, were diluted 100 times with additional osmotic buffer (10 mM HEPES, $100 \mathrm{mM}$ $\mathrm{NaCl}, \mathrm{pH}$ 7.4). $15 \mu \mathrm{L}$ of sample were placed then for 1 minute on a copper grid coated with a formvar carbon film. The excess of sample was wicked away with the aid of filter paper, and the sample was stained with $15 \mu \mathrm{L}$ of Uranyl Acetate (1\%) for $3 \mathrm{~min}$. The excess of staining solution was wicked away with the aid of filter paper and the sample was allowed to dry overnight prior to imaging by TEM. Measurements quoted are the averages of at least 100 particles.

\section{Zeta potential}

Polymeric vesicles prepared as described above, were filtered through $0.2 \mu \mathrm{m}$ syringe filter and diluted with additional osmotic buffer (10 mM HEPES, $100 \mathrm{mM} \mathrm{NaCl}, \mathrm{pH}$ 7.4). The values reported are the average of 3 measurements.

\section{Vesicle stability}

$20 \mu \mathrm{L}$ of polymeric vesicle solution prepared as described above, containing $50 \mathrm{mM} \mathrm{CF}$, were diluted up to $1 \mathrm{~mL}$ with additional osmotic buffer. The time dependent change in fluorescence intensity $\left(I_{\mathrm{t}}\right)\left(\lambda_{\text {exc }}=492 \mathrm{~nm}, \lambda_{\mathrm{em}}=517 \mathrm{~nm}\right)$ was monitored for at least $1 \mathrm{~h}$ in the case of $\mathrm{pH}$ stability, and at least overnight in the case of buffer stability. Encapsulated dye was released by disruption of the vesicle membrane as described above with Triton X-100. Time courses of $I_{\mathrm{t}}$ were normalised to relative intensity $\left(I_{\mathrm{R}}\right)$ using the following equation (eqn (2)),

$$
I_{\mathrm{R}}=\left(I_{\mathrm{t}}-I_{0}\right) /\left(I_{\mathrm{T}}-I_{0}\right) \times 100,
$$

where $I_{0}=I_{\mathrm{t}}$ at the beginning of the experiment and $I_{\mathrm{T}}=I_{\mathrm{t}}$ after lysis with Triton X-100.

\section{Uptake}

3T3 Fibroblasts or A549 cells were grown to a confluent monolayer in tissue culture treated 96 well plates. The cells were then incubated with a growth media, or media without FBS for b-PEI coated vesicles, containing polymeric vesicles $\left(3 \mathrm{mg} \mathrm{mL}^{-1}\right)$ loaded with $50 \mu \mathrm{M} \mathrm{CF}$. The cells were incubated with the vesicles for up to 72 hours. At regular time point throughout the incubation the media was removed, the cells washed with PBS and fluorescent images were acquired. Uptake was measured via analysis of intensity of the green channel in the images.

\section{Cytotoxicity assays}

Acute toxicity was measured via the LDH assay. Media removed from each well prior to the PBS washes for imaging was assessed for LDH activity using the CytotoxONE ${ }^{\mathrm{TM}}$ assay kit, whereby non fluorescent resazurin is reduced to resorufin in the presence of LDH. Plates containing the supernatant of the treated cells 
were incubated in the presence of the LDH reagent for $15 \mathrm{~min}$ at room temperature and the reaction stopped using the STOP solution. Fluorescence intensity $\left(\lambda_{\text {exc }}=560 \mathrm{~nm}, \lambda_{\text {em }}=590 \mathrm{~nm}\right)$ was then measured compared to the fluorescence intensity of an untreated control (spontaneous LDH release) and cells treated with 4\% Triton X-100 (100\% LDH release).

Chronic toxicity was measured via the MTT assay. Cells which had previously been treated with the vesicles were incubated for a further 24 hours in growth media, washed with PBS, and incubated for a further 2 hours with growth media containing $1 \mathrm{mg}$ $\mathrm{mL}^{-1}$ MTT reagent. The cells were then washed once with PBS and $300 \mu \mathrm{L}$ DMSO was added to each well and the plates incubated for $30 \mathrm{~min}$ to allow the formazan product to dissolve completely. $A_{490}$ was measured for each treatment, and compared to untreated cells (100\% MTT metabolism) and cells treated with 4\% Triton X-100 ( $0 \%$ MTT metabolism).

\section{Results}

In order to produce robust vesicles that could withstand different aqueous conditions, acrylamide monomers were chosen. Amides have a higher stability against hydrolysis when compared to esters such as those in acrylates and methacrylates. In addition, a hydrophobic backbone with high crystallinity was desired, as it will provide a tighter packing than aliphatic residues, such as those in biologically relevant lipids. This way the mechanical properties of the vesicles will be improved leading to a higher resistance of the membrane to the diffusion of small molecules. With these considerations in mind, phenylacrylamide (PAm) and $N$ - $\omega$-acrylamidobutanoic acid (4AmBA) were chosen as the hydrophobic and hydrophilic components. Both monomers were prepared in good yields and high purity by modification of a previously reported protocol $(\mathrm{ESI} \dagger){ }^{19}$

To synthesise well defined polymers that could be chain extended into the desired amphiphilic blocks, controlled living polymerisation (CLP) techniques were required. From the currently available CLP, RAFT polymerisation is probably the most versatile and suitable for the preparation of acrylamide based polymers. ${ }^{22-26}$ The polymerisation of PAm using CTA1 as the RAFT agent and $\mathbf{V - 5 0 1}$ as the radical source, in DMF at $70{ }^{\circ} \mathrm{C}$ proved to be well controlled. As expected for any free radical polymerisation, the reaction followed pseudo-first order

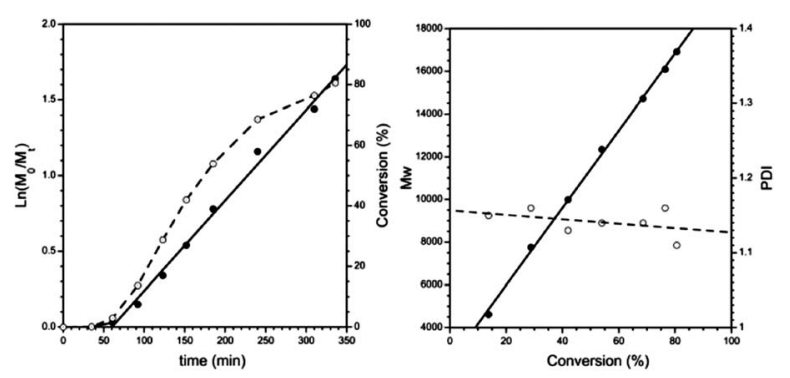

Fig. 1 Left: representative linear plot of $\ln [\mathrm{M}]_{0} /[\mathrm{M}]_{\mathrm{t}} v s$. time (solid) and plot of conversion vs. time (dashed). Right: representative plot of measured MW vs. conversion (solid) and PDI vs. conversion (dashed). Conditions: $70{ }^{\circ} \mathrm{C} ;[\mathbf{P A m}]=1.6 \mathrm{M} ;[\mathbf{C T A 1}] /[\mathbf{P A m}]=100 ;[\mathbf{C T A 1}] /[\mathbf{V}-\mathbf{5 0 1}]$ $=3$; DMF.

kinetics with a small induction period of approximately 30-40 min, typical of RAFT polymerisations. In addition there was a linear increase of MW with conversion while the polydispersity of the material was narrow throughout the polymerisation (Fig. 1). In this way, several materials could be prepared, with different MW (Table 1, entries 1-3, P1).

For the polymerisation of $\mathbf{A A m B A}$, the same conditions were initially investigated. However, monomer conversion was very low, even at the high concentrations employed (1.6 M). Several conditions were surveyed, that involved different solvents $\left(\mathrm{H}_{2} \mathrm{O}\right.$, $\left.\mathrm{H}_{2} \mathrm{O}-\mathrm{EtOH}\right)$ or the use of different RAFT agents. Interestingly, by employing trithiocarbonates CTA2 or CTA3, the polymerisations proceeded much faster. Once again, pseudo-first order kinetics was observed with good control both over the MW and the polydispersity of the materials obtained (Fig. S06, ESI $\dagger$ ). In a similar fashion to PAm, a series of polymers with different MW were prepared (Table 2, entries 5-7, P2).

For the preparation of amphiphilic block copolymers, the chain extension of p(PAm) (P1) was initially investigated. The choice of solvent proved to be complicated, due to the different solubility of both blocks. P1 are highly insoluble in the presence of water but can be solubilised in organic solvents such as alcohols, DMSO and DMF depending on their concentration and MW. P2, on the other hand, were only soluble in DMSO, low MW alcohols or aqueous solutions, with solubility in DMF strongly depending on their MW. Initially, the same conditions employed for the preparation of $\mathbf{P 1}$ were investigated (Table 1,

Table 1 Polymers described in this paper

\begin{tabular}{|c|c|c|c|c|c|c|c|c|c|c|}
\hline Entry & $\mathrm{P}$ & Solvent & CTA & {$[\mathrm{M}]$} & Time $\min$ & $c(\%)$ & Final DP & Mn (NMR) & Mn (GPC) & PDI \\
\hline 1 & P1a & DMF & CTA1 & 1.60 & 944 & 85 & 22 & 3420 & $7309^{b}$ & 1.35 \\
\hline 2 & P1b & $\mathrm{DMF}$ & CTA1 & 1.60 & 944 & 85 & 42 & 6404 & $13127^{b}$ & 1.37 \\
\hline 3 & Plc & DMF & CTA1 & 1.60 & 1020 & 84 & 66 & 9802 & $11575^{b}$ & 1.18 \\
\hline 4 & Pld & DMF & CTA2 & 0.81 & 205 & 76 & 42 & 6382 & $12330^{b}$ & 2.09 \\
\hline 5 & $\mathrm{P} 2 \mathrm{a}$ & $\mathrm{H}_{2} \mathrm{O}^{a}$ & CTA3 & 0.75 & 181 & 92 & 28 & 4572 & $3492^{c}$ & 1.09 \\
\hline 6 & $\mathrm{P} 2 \mathrm{~b}$ & $\mathrm{H}_{2} \mathrm{O}^{a}$ & CTA3 & 0.47 & 150 & 88 & 43 & 7006 & $13935^{c}$ & 1.06 \\
\hline 7 & P2c & $\mathrm{H}_{2} \mathrm{O}^{a}$ & CTA3 & 0.74 & 60 & 96 & 94 & 14985 & $19812^{c}$ & 1.19 \\
\hline 8 & $\mathrm{P} 3 \mathrm{a}$ & DMF & Plc & 1.60 & 625 & 38 & 14 & 11946 & $17413^{b}$ & 1.20 \\
\hline 9 & P3b & Dioxane & P1b & 1.59 & 960 & 57 & 16 & 8896 & - & - \\
\hline 10 & $\mathrm{P} 3 \mathrm{c}$ & $\mathrm{DMSO}^{a}$ & $\mathrm{P} 2 \mathrm{a}$ & 0.50 & 72 & 88 & 87 & 17245 & - & - \\
\hline 11 & P3d & $\mathrm{DMSO}^{a}$ & $\mathrm{P} 2 \mathrm{a}$ & 0.50 & 77 & 86 & 78 & 21481 & - & - \\
\hline 12 & $\mathrm{P} 3 \mathrm{e}$ & DMSO & $\mathrm{P} 2 \mathrm{a}$ & 0.50 & 70 & 57 & 31 & 9198 & - & - \\
\hline 13 & P3f & DMSO & $\mathrm{P} 2 \mathrm{a}$ & 0.50 & 36 & 33 & 18 & 7225 & - & - \\
\hline
\end{tabular}

${ }^{a}$ CTA and V-501 added in EtOH. ${ }^{b}$ Measured using DMF as the eluent. ${ }^{c}$ Measured using DPBS as the eluent. 

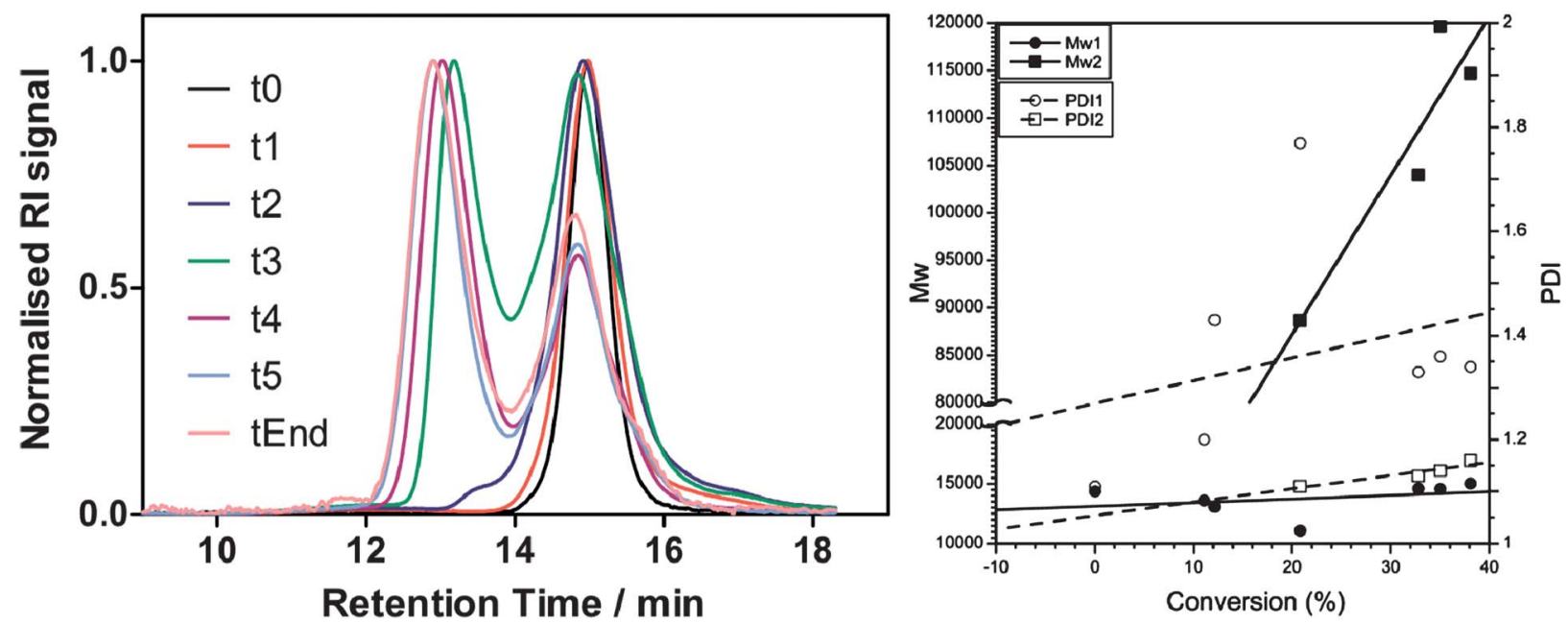

Fig. 2 Left: GPC traces and Right: plot of measured MW vs. conversion and PDI $v s$. conversion for the two populations observed during the polymerisation of 4AmBA using P1c as the macro-RAFT agent to yield P3a.

entry 8, P3a). Polymerisations proceeded slowly, and only $38 \%$ of conversion could be achieved after more than $10 \mathrm{~h}$, in agreement with the low reactivity of $\mathbf{A A m B A}$ under these conditions (Fig. S09, ESI $\dagger$ ).

The incompatible solubility of both blocks was also problematic for the analysis of the final materials. THF, $\mathrm{CHCl}_{3}$ or aqueous conditions could not be employed for the GPC analysis, and only DMF/0.1\% $\mathrm{LiBr}$ seemed to be able to solubilise both blocks. However, the presence of two populations was detected after $20 \%$ of conversion was achieved. We infer that not all of the available starting blocks were able to initiate the polymerisation and give amphiphilic blocks, and those that did gave very high MW species (Fig. 2).

This behaviour was consistent with the macro-RAFT agents showing a slow initiation rate while the polymerisation proceeded with a high propagation rate. On the other hand, the hydrophilic block was sparingly soluble in DMF, and DLS analysis indicated that P3a might have aggregated in DMF. Therefore P3a, while dissolved in $\mathrm{DMF} / 0.1 \% \mathrm{LiBr}$, could be forming colloidal aggregates, leading to materials with very high apparent MW when measured by GPC. If this were the case, the degree of aggregation would be affected by dilution and salt concentrations. Interestingly, the relative intensity of the two populations observed in the GPC was strongly dependent on both factors (Fig. 3), suggesting that aggregation was interfering with the characterisation.

In an attempt to improve the solubility of the hydrophilic block in DMF, the acid groups were esterified with EtOH. In all cases the percentage of the higher MW population was significantly reduced, supporting the idea that aggregation during GPC analysis could be compromising the characterisation of these amphiphilic block copolymers (Fig. S10, ESI $\dagger$ ).

The polymerisation of $\mathbf{4 A m B A}$ with $\mathbf{P 1}$ as macroRAFT agents was also tested using different solvents, such as dioxane, but similar results were obtained, with 2 populations observed in the GPC. In addition, since trithiocarbonates were more efficient for the polymerisation of 4AmBA, the polymerisation of PAm with CTA2 as the RAFT agent was also investigated, with the aim of preparing a better macro-RAFT agent for the synthesis of
(PAm)-b-(4AmBA) amphiphiles. Unfortunately, control over the polymerisation was lost in this case as reflected by the high PDI of the final material (Table 1, entry 4, P1d).

When the desired amphiphiles were prepared by chain extension of P2 using PAm as the monomer, similar results were obtained (Table 1, entries 10-13, P3c-f). Again, pseudo-first order kinetics was observed, but the polymerisation proceeded in shorter times, in relation to the faster kinetics observed so far with the trithiocarbonates (Fig. S09, ESI $\dagger$ ). When analysed by GPC, again two populations could be detected.

While control over the polymerisation is desirable for the preparation of well-defined vesicles, it is not a key element. The MW of the polymer is important for vesicle formation, but it is expected that amphiphiles with a broad range of MW should be able to segregate the longer chains to the outer side of the vesicle while shorter polymers will accommodate in the inside. ${ }^{27}$ On the other hand, the most important parameter in order to predict if an amphiphile will form vesicular aggregates in solution is the volume fraction of the hydrophilic block. Volume fraction can be difficult to calculate, especially in the absence of ideal solvents for both blocks. Alternatively it has been estimated that for those amphiphiles having weight fractions of the hydrophilic block between 25 and $35 \%$, a vesicle in aqueous solution could be expected.$^{28}$ In order to investigate the best architecture for vesicle
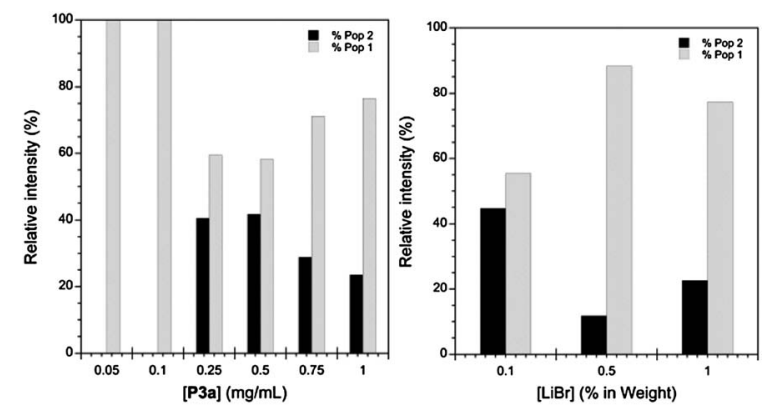

Fig. 3 Left: percentage of the two populations observed in the GPC as a function of P3a concentration. Right: percentage of the two populations observed in the GPC as a function of $\mathrm{LiBr}$ concentration. 
Table 2 Characterisation of colloidal aggregates in aqueous conditions for P3

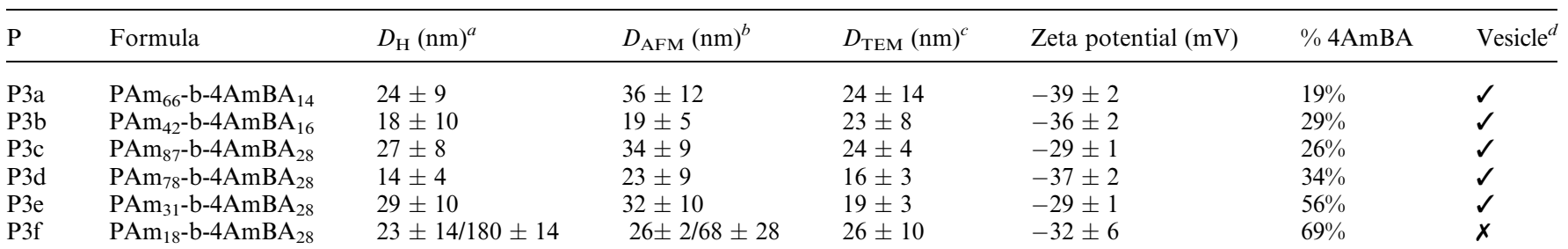

${ }^{a}$ Measured in HEPES buffer (10 mM, $100 \mathrm{mM} \mathrm{NaCl}, \mathrm{pH}$ 7.4) by DLS. Number distribution. ${ }^{b}$ Measured in HEPES buffer (10 mM, $100 \mathrm{mM} \mathrm{NaCl,} \mathrm{pH}$ 7.4) by AFM. ${ }^{c}$ Measured over dry samples by TEM. ${ }^{d} \checkmark$ Denotes a positive result, where a significant increase in fluorescence was observed after addition of Triton, $\boldsymbol{X}$ denotes a negative result where no increase or reduction of fluorescence was observed after addition of Triton. 5-10 mg mL ${ }^{-1}$ of $\mathrm{P} 3$ for vesicle preparation.

formation, we decided to prepare a series of polymers ranging hydrophilic weight percentage between 20 and 60\% (Table 1, entries 7-14, P3). This weight percentage was estimated from the monomer composition measured by NMR. In all cases, the RAFT agent was removed by treatment with an excess of azo initiator, ${ }^{29}$ in order to prevent any side reaction derived from its cleavage.

Two main protocols were investigated for vesicle preparation: (a) a film method, in analogy to most of the work done with vesicles made from standard lipids and amphiphiles; and (b) a solvent exchange method, widely applied for the preparation of polymersomes. In our hands, the film method was less reliable, as the amphiphiles precipitated upon resuspension in the vesicle buffer, with no apparent relation between amphiphile concentration or formulation. On the other hand, the solvent exchange method, where the polymers were dissolved in a good volatile solvent that could be added onto vesicle buffer and left to evaporate overnight, gave better and more consistent results.

In order to confirm if the aggregates formed using these two protocols contained a hydrophilic core, a dye encapsulation method was employed. CF was chosen as a model hydrophilic dye, which should be encapsulated within the aqueous core of the vesicles. Therefore, $50 \mathrm{mM} \mathbf{C F}$ solutions, a concentration at which $\mathbf{C F}$ is self-quenched, were employed as the buffer for vesicle formation. The excess of dye was removed via extended dialysis against osmotic buffer (HEPES $10 \mathrm{mM}, \mathrm{NaCl} 100 \mathrm{mM}$, $\mathrm{pH} 10$ and 7.4), and the encapsulation of CF confirmed by means of fluorescence (Fig. 4, Table 2). As expected, almost no fluorescence was observed when the vesicles were diluted 500 fold in the osmotic buffer, as the concentration of the dye should remain at $50 \mathrm{mM}$ within the vesicles. When vesicles were burst with a surfactant, release of the encapsulated dye led to a significant increase in fluorescence, as the original self-quenching conditions no longer apply. It is worth noting that most of the block copolymer prepared in this work lead to the formation of vesicular aggregates (Table 2), suggesting that for these materials, the ratio of hydrophilic weight percentage suitable for vesicle formation was bigger than $25-35 \%$.

The characterisation of the vesicles by means of DLS revealed that the sizes of the aggregates in solution were, in most of the cases, below $100 \mathrm{~nm}$ in diameter, with very low polydispersities (Table 2). In order to have a better insight into the shape and morphology in solution of these vesicles, we used AFM analysis using PeakForce tapping mode. AFM analysis allows imaging in a liquid environment and this newly available imaging mode enables greater control of the probe-sample contact force when compared to tapping mode, and materials can be studied in liquid environment. Accordingly, artefacts, such as those resulting from drying, can be avoided.

AFM revealed that in all cases spherical objects were observed with sizes in close agreement with those measured by DLS (Fig. 5, Table 2), only slightly higher diameters were measured by AFM. This difference may arise from the fact that when calculating number distributions by DLS, the population of smaller objects tends to be overestimated. In addition, the features measured by AFM are usually a bit broader than the measured diameter by DLS, as a result of the finite size of the apex of the AFM probe.

In order to confirm the spherical morphology of these aggregates, they were analysed using negative staining TEM. In this case, the sizes measured by TEM were slightly smaller than those measured by AFM. This reduction in size is expected, as the drying of the materials will lead to a collapse of the hydrophilic corona, which should be fully swelled in aqueous conditions. On the other hand, this drying did not have a strong impact on the integrity of the aggregates, and in all cases, spherical objects could be observed. In addition, TEM suggested that the aggregates prepared with P3a-e were hollow structures while for P3f micelles seemed to be the most common morphology (Fig. 5 and Fig. S12, ESI $\dagger$ ).

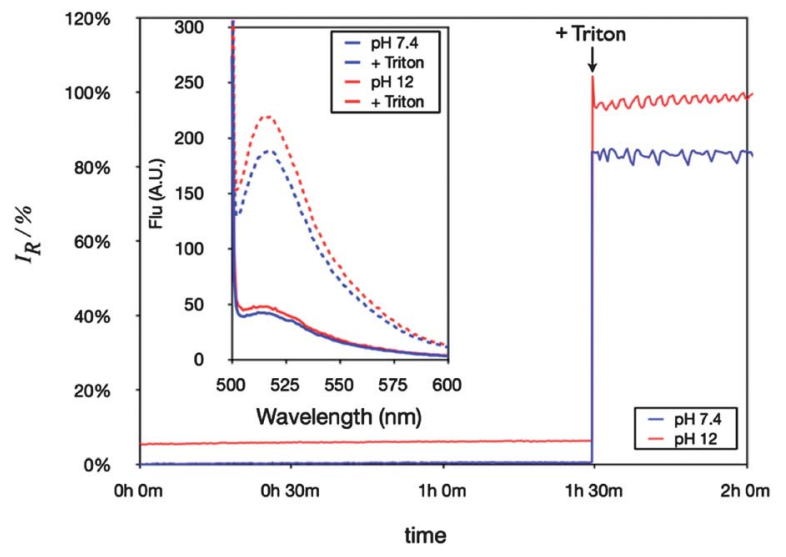

Fig. 4 Evolution of fluorescence as a function of time, measured at $\mathrm{pH}$ 7.4 and 12. Inset: Representative fluorescence profiles of $\mathbf{C F}$ encapsulated in vesicles (P3b $5 \mathrm{mg} \mathrm{mL}^{-1}$ employed in this example, vesicles were burst after $1 \mathrm{~h} 30 \mathrm{~min}$ by addition of Triton). 


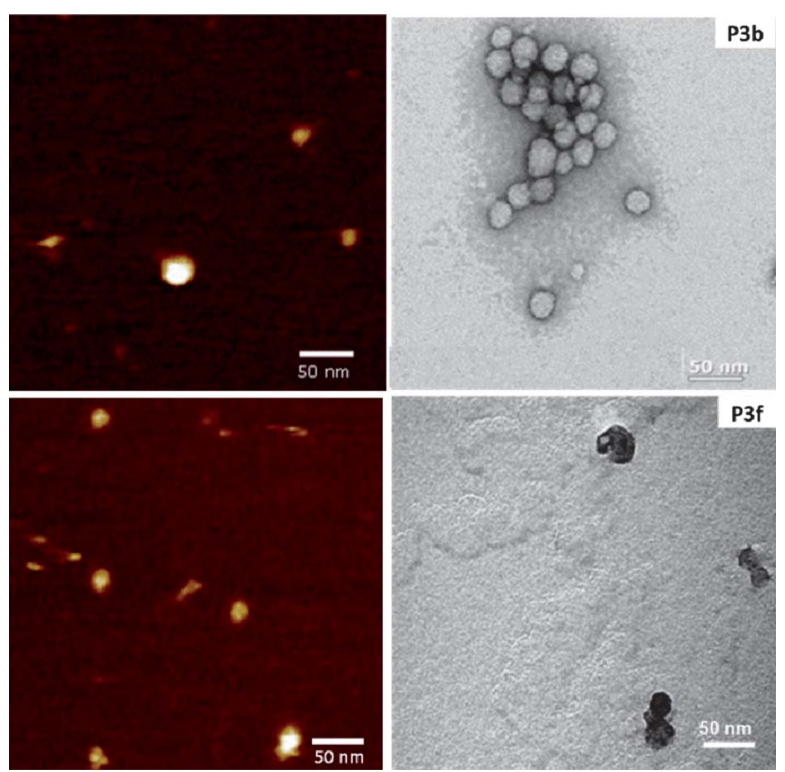

Fig. 5 Representative examples of vesicle morphology as seen by AFM in solution (left) and negative staining TEM (right) (for AFM images vertical scales are $4 \mathrm{~nm}$ ). $10 \mathrm{mg} \mathrm{mL}^{-1}$ of $\mathbf{P 3}$ for vesicle preparation.

Zeta potentials of the particles indicated, as expected, that particles were negatively charged, due to their carboxylic acid end groups.

Having prepared several amphiphilic block copolymers that assembled into spherical vesicles in solution, we were interested in determining stability under different aqueous conditions. CF-loaded vesicles were stable at neutral $\mathrm{pH}$, with no apparent release of the dye for at least $1 \mathrm{~h}$ (Fig. 4, blue line). In a similar fashion, when those vesicles were placed in the same osmotic buffer but at a much higher $\mathrm{pH}$, still no release of the vesicle contents could be observed (Fig. 4, red line). DLS analysis showed that almost no difference in size could be detected for the vesicles at both $\mathrm{pH}$ values (Fig. S13, ESI†).

Vesicle stability was also analysed by dilution into osmotic buffers containing different salts and ionic strengths. When $\mathbf{C F}$ loaded vesicles were diluted with an osmotic buffer containing $100 \mathrm{mM} \mathrm{NaCl}$, the one used as a default osmotic buffer, only $15 \%$ of the $\mathbf{C F}$ was released after incubating overnight at room temperature (Fig. 6). Similar release of $\mathbf{C F}$ was observed when different ionic strengths were employed $(\mathrm{NaCl}, 10 \mathrm{mM}$ and $1 \mathrm{M}$, Fig. 6) while no significant increase was observed when the vesicles were left to incubate for longer periods of time, even up to 4 days (Fig. S14, ESI†). This lack of further release after the first $24 \mathrm{~h}$ suggests that the initial release was probably due to $\mathbf{C F}$ loosely bound to the hydrophilic corona of the vesicles. Interestingly, similar levels of release were observed when other salts where employed such as $\mathrm{Na}_{2} \mathrm{CO}_{3}, \mathrm{KCl}$ or $\mathrm{CaCl}_{2}$. Only in the case of $1 \mathrm{M} \mathrm{Na}_{2} \mathrm{CO}_{3}$, was the amount of dye released notably different, being increased to almost $40 \%$ of the initial $\mathbf{C F}$ content (Fig. S14, ESI $\uparrow$ ). In all cases, DLS indicated that the size of the particles had not changed significantly after this incubation, and that the vesicles were stable in the presence of a wide range of salts under different ionic strengths (Fig. S15, ESI $\dagger$ ).

The stability of these vesicles was in sharp contrast to that of liposomes prepared from 1,2-dioleoyl-sn-glycero-3-phospho-rac- (1-glycerol) sodium salt (DOPG), a model lipid that is also negatively charged in aqueous solutions. As expected, DOPG liposomes showed improved stability with increasing ionic strength. On the other hand, when $\mathrm{NaCl}$ was substituted for other salts, DOPG vesicles proved to be more unstable and for example more than $50 \%$ of the dye was released in the presence of $1 \mathrm{M} \mathrm{CaCl}_{2}$ (Fig. 6).

To test the biocompatibility of these materials, polymersomes were loaded with $50 \mu \mathrm{M} \mathrm{CF}$ and incubated with 3T3 fibroblasts and A549 cells; 3T3 fibroblasts represent a spontaneously immortalised embryonic fibroblast cell line while A549 cells represent an epithelial lung tumour cell line. These specific cell lines were chosen as difference in uptake of liposomal complexes has been reported by normal and tumour derived cell lines. ${ }^{14,30}$ Uptake of the vesicles was observed in both cell lines, without significant acute or chronic toxicity, up to 72 hours. However the uptake was slow, taking 16 hours to saturate in $3 \mathrm{~T} 3$ fibroblasts (Fig. 7). This slow uptake was expected due to the repulsive forces between the negative surface of the vesicles and the negatively charged outer leaflet of the cell membrane. ${ }^{31}$

In order to improve uptake, a simple coating protocol, for which polymersomes solutions were mixed with b-PEI, was employed. Using this simple step, the charge on the surface of the vesicles was inverted (zeta potential $41 \pm 2 \mathrm{mV}$ ). To minimise polymersome membrane disruption by b-PEI (Fig. S16, ESI $\dagger$ ), an excess of this polymer was employed. After coating with b-PEI, vesicles could be imaged by AFM, using now negatively charged freshly cleaved mica. The spherical shape of the particles was not affected, and no aggregation could be observed (Fig. 7). In addition, uptake was significantly enhanced, saturating after about $30 \mathrm{~min}$ in A549 cells, at the expense of biocompatibility. Polymersomes modified with b-PEI caused $25 \%$ cell lysis after a 30 min incubation and $100 \%$ following a 60 min incubation, as determined by the LDH release assay. When chronic toxicity was measured 24 hours post incubation, only $18 \%$ of the cells remained metabolically active following a 30 min incubation. Cytotoxicity was reduced when a 15 min incubation was employed, and $90 \%$ of the cells remained metabolically active, as

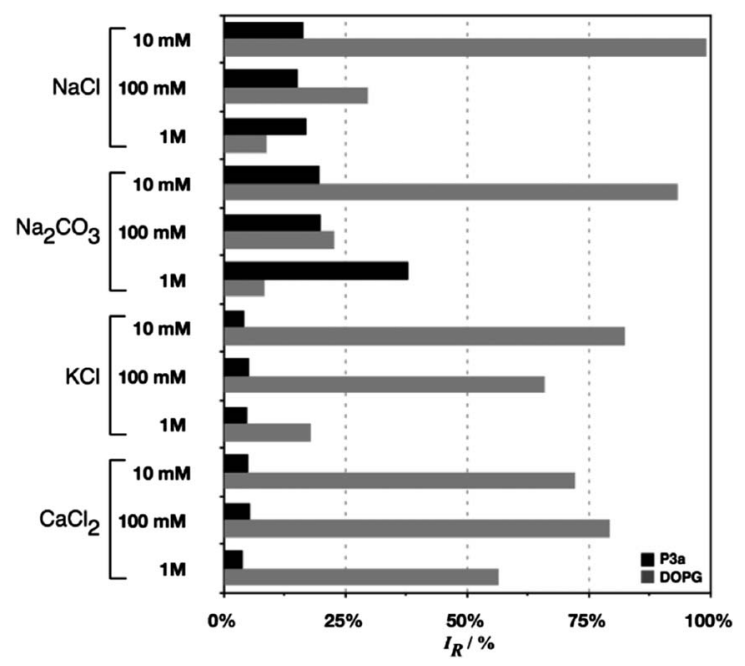

Fig. 6 Percentage of $\mathrm{CF}$ released after incubating vesicles o.n. in the presence of different osmotic buffers containing different salts and ionic strengths. $5 \mathrm{mg} \mathrm{mL}^{-1}$ of $\mathbf{P} \mathbf{3 b}$ for vesicle preparation. 

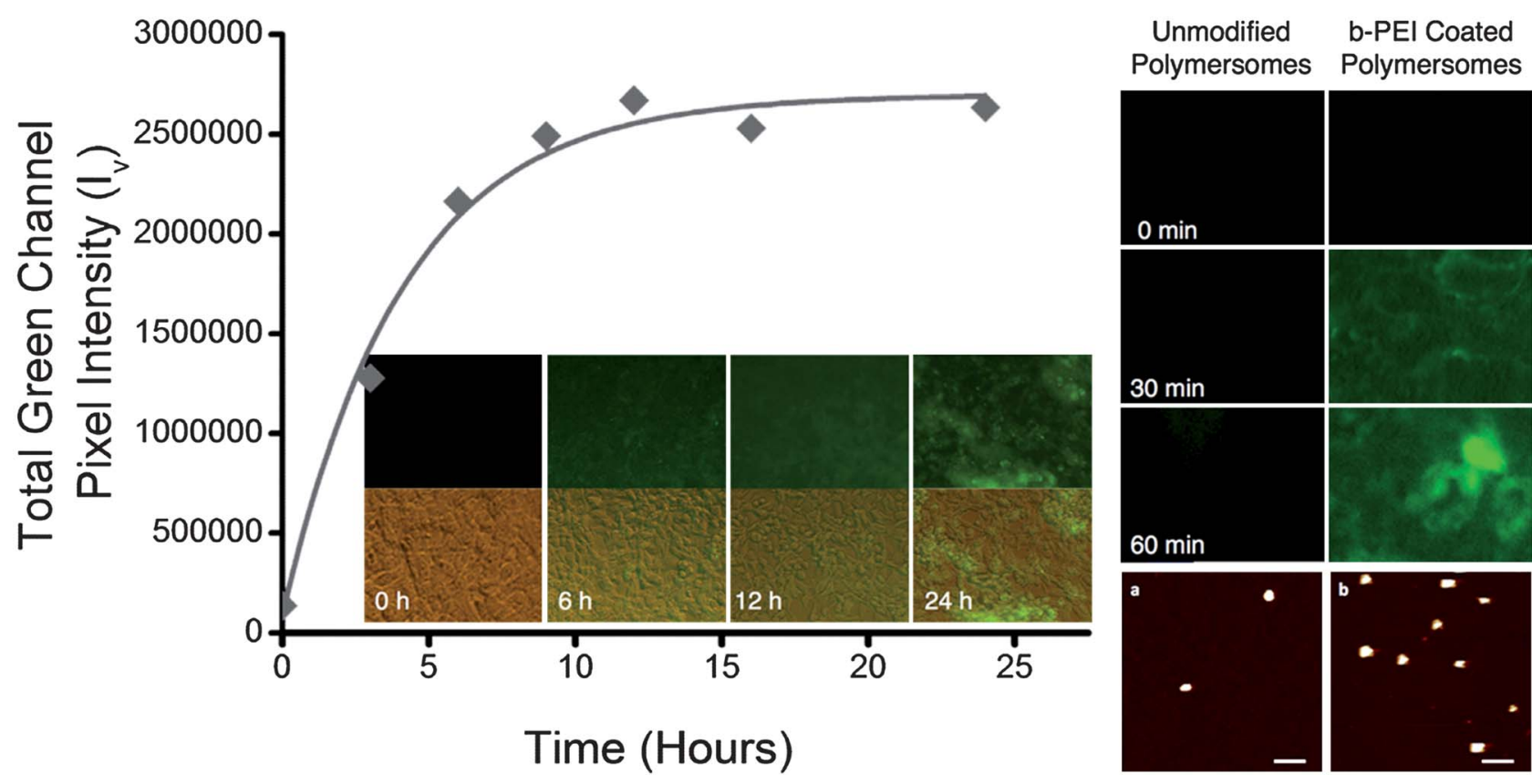

Fig. 7 Left: uptake of polymersomes by 3 T3 fibroblast and representative fluorescent (top) and merged (bottom) micrographs. Right: fluorescent micrographs of uptake by A549 cells of unmodified $v$ s. b-PEI coated polymersomes, and representative example of vesicle morphology by AFM before (a) and after (b) coating with b-PEI. Scale bars: $200 \mathrm{~nm}$, vertical scales: $20 \mathrm{~nm}$.

determined by the MTT assay. The acute toxicity is more than likely caused by the membrane disruptive effects of b-PEI, as can be observed from the brightfield images of the cells following a 60 min incubation (Fig. S17, ESI†). This is in sharp contrast with the stability of the polymeric vesicles, which were able to stand this concentration of $\mathbf{b}$-PEI without significant changes to their morphology (Fig. 7) or the release of the loaded CF. The increase between acute and chronic toxicity in the 30 min incubation can be accounted for by the destabilisation of organelle membranes by $\mathbf{b}$-PEI and the subsequent trigger of both apoptotic and necrotic cell death. ${ }^{32}$ Due to the greatly enhanced uptake of the bPEI modified polymersomes, much smaller concentrations of polymersomes could be employed in further experiments to extend the incubation window at which the polymersomes are biocompatible whilst still maintaining rapid saturation of polymersome uptake.

\section{Conclusions}

In conclusion, a series of polymeric amphiphiles with the general structure $\mathbf{p}(\mathbf{4 A m B A})-\mathbf{b}-(\mathbf{P A m})$ have been prepared. Those amphiphiles with percentages of hydrophilic monomer between 20 and $55 \%$ in weight, self assemble in aqueous environment to yield well defined polymersomes. These vesicular aggregates have been characterised by means of DLS, AFM and TEM, showing that in most cases the size and shape of the aggregates obtained were well defined, with sizes ranging $20-30 \mathrm{~nm}$ and in very narrow size distributions. The ability of these polymersomes to encapsulate hydrophilic molecules was evaluated using CF. The polymersomes proved to be remarkably stable across a range of conditions such as increased $\mathrm{pH}$, in the presence of different buffer strengths and regardless of the salt nature. This stability is in sharp contrast to those vesicles prepared with DOPG. In addition, the vesicles are biocompatible, showing no toxicity towards 3T3 and A549 cell lines, and their cell uptake can be improved by a simple coating protocol. Alternatively, decoration of the surface of the vesicles with targeting ligands via covalent linkages could also lead to improved uptake. All of these properties suggest that polymersomes of this type may find potential applications in fields such as materials science, diagnostics and imaging.

\section{Acknowledgements}

We thank the UK EPSRC and BBSRC (Grants EP/G042462/1, EP/D022347/1, D021847/1 and BB/F01855X/1) and the University of Nottingham, U.K., for funding. We thank Dr Jonathan Aylott (University of Nottingham) for access to Fluorometer, Dr Chis Parmenter and Dr Emma King (University of Nottingham) for help with TEM imaging.

\section{Notes and references}

1 F. Meng, Z. Zhong and J. Feijen, Biomacromolecules, 2009, 10, $197-$ 209.

2 O. Onaca, R. Enea, D. W. Hughes and W. Meier, Macromol. Biosci., 2009, 9, 129-139.

3 W. T. Al-Jamal and K. Kostarelos, Acc. Chem. Res., 2011, 44, 1094 1104.

4 R. P. Brinkhuis, F. P. J. T. Rutjes and J. C. M. van Hest, Polym. Chem., 2011, 2, 1449-1462.

5 K. Renggli, P. Baumann, K. Langowska, O. Onaca, N. Bruns and W. Meier, Adv. Funct. Mater., 2011, 21, 1241-1259.

6 U. J. Meierhenrich, J.-J. Filippi, C. Meinert, P. Vierling and J. P. Dworkin, Angew. Chem., Int. Ed., 2010, 49, 3738-3750.

7 P. Walde, K. Cosentino, H. Engel and P. Stano, ChemBioChem, 2010, $1-19$.

8 B. M. Discher, Y. Y. Won, D. S. Ege, J. C. Lee, F. S. Bates, D. E. Discher and D. A. Hammer, Science, 1999, 284, 1143-1146. 
9 J. C. Lee, H. Bermudez, B. M. Discher, M. A. Sheehan, Y. Y. Won, F. S. Bates and D. E. Discher, Biotechnol. Bioeng., 2001, 73, 135145.

10 H. Bermudez, A. Brannan, D. Hammer, F. Bates and D. Discher, Macromolecules, 2002, 35, 8203-8208.

11 M. Antonietti and S. Forster, Adv. Mater., 2003, 15, 1323-1333.

12 B. Discher, D. Hammer, F. Bates and D. Discher, Curr. Opin. Colloid Interface Sci., 2000, 5, 125-131.

13 D. E. Discher and A. Eisenberg, Science, 2002, 297, 967-973.

14 P. L. Soo and A. Eisenberg, J. Polym. Sci., Part B: Polym. Phys, 2004, 42, 923-938.

15 T. Smart, H. Lomas, M. Massignani, M. V. Flores-Merino, L. R. Perez and G. Battaglia, Nano Today, 2008, 3, 38-46.

16 A. Blanazs, S. P. Armes and A. J. Ryan, Macromol. Rapid Commun., 2009, 30, 267-277.

17 J. Du and R. K. O'reilly, Soft Matter, 2009, 5, 3544-3561.

18 C. Lopresti, H. Lomas, M. Massignani, T. Smart and G. Battaglia, J. Mater. Chem., 2009, 19, 3576.

19 J. Auernheimer, C. Dahmen, U. Hersel, A. Bausch and H. Kessler, J. Am. Chem. Soc., 2005, 127, 16107-16110.

20 S. Thang, Y. Chong, R. Mayadunne, G. Moad and E. Rizzardo, Tetrahedron Lett., 1999, 40, 2435-2438.

21 J. Skey and R. K. O'reilly, Chem. Commun., 2008, 4183-4185.
22 K. Matyjaszewski, in Controlled/Living Radical Polymerization: Progress in ATRP, ed. K. Matyjaszewski, American Chemical Society, Washington, DC, 2009, vol. 1023, pp. 3-13.

23 G. Moad, E. Rizzardo and S. Thang, Aust. J. Chem., 2004, 58, 379 410.

24 A. Lowe and C. McCormick, Prog. Polym. Sci., 2007, 32, 283-351.

25 C. Barner-Kowollik, Handbook of RAFT Polymerization, WileyVCH, Weinheim, 2008.

26 C. Boyer, V. Bulmus, T. Davis, V. Ladmiral, J. Liu and S. Perrier, Chem. Rev., 2009.

27 O. Terreau, L. Luo and A. Eisenberg, Langmuir, 2003, 19, 5601-5607.

28 D. E. Discher and F. Ahmed, Annu. Rev. Biomed. Eng., 2006, 8, $323-$ 341.

29 S. Perrier, P. Takolpuckdee and C. Mars, Macromolecules, 2005, 38, 2033-2036.

30 I. Ito, G. Began, I. Mohiuddin, T. Saeki, Y. Saito, C. D. Branch, A. Vaporciyan, L. C. Stephens, N. Yen, J. A. Roth and R. Ramesh, Mol. Ther., 2003, 7, 409-418.

31 C. R. Miller, B. Bondurant, S. D. McLean, K. A. McGovern and D. F. O'Brien, Biochemistry, 1998, 37, 12875-12883.

32 O. M. Merkel, A. Beyerle, B. M. Beckmann, M. Zheng, R. K. Hartmann, T. Stöger and T. H. Kissel, Biomaterials, 2011, 32, 2388-2398. 